\title{
Lo que el arte \\ puede enseñar al \\ diseño industrial: una \\ experiencia de taller a \\ partir del análisis de \\ " el entierro del Conde de \\ Orgaz"
}

What art can teach in industrial design: a workshop experience from the analysis of "the burial of the count of Orgaz"

VÍCTOR A. LAFUENTE - DANEL LÓPEZ - MARÍA VARELA valszb@hotmail.com·daniel.lopez.bragado@uva.es·maria.varela.ulloa@alumnos.uva.es

Universidad de Valladolid 
$\Rightarrow$ Recibido 28/10/2018

Aceptado 10/01/2019

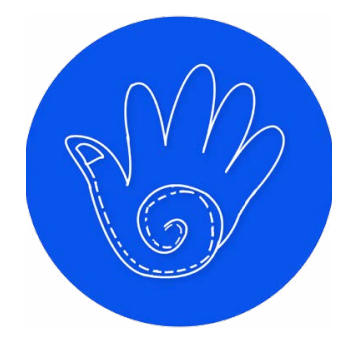

\section{Resumen}

La llegada del Espacio Europeo de Educación Superior ha influido en el modo de desarrollar la docencia de las disciplinas gráficas en los estudios de Ingeniería en Diseño Industrial en nuestro país. Uno de los cambios más relevantes ha representado la implantación en las aulas del formato de taller, especialmente en las asignaturas de corte artístico; la intención última es que el alumno adquiera, de manera práctica, las competencias específicas relacionadas con su capacitación para la comunicación simbólica y la profundización en el análisis gráfico del mundo que le rodea. Este proceso obliga a una permanente síntesis de las formas, los colores y las luces de los objetos, para ser plasmados mediante las destrezas y métodos gráficos que adquieran a lo largo de toda su vida académica. Se pretende aquí ejemplificar este proceso con el estudio y análisis gráfico de una obra de arte, mostrando la relación existente entre el Diseño Industrial y la docencia humanística. Para ello se ha elegido la obra“El Entierro del Conde de Orgaz", pintado por El Greco, en el que reside una gran complejidad compositiva, llena de contrastes e intencionalidad, pero a la vez enormemente didáctica desde el punto de vista plástico. 


\begin{abstract}
The arrival of the European Higher Education Area has influenced the way to develop the teaching of the graphic disciplines in Industrial Design Engineering studies in our country. One of the most relevant changes has been the introduction of the workshop format in the classrooms, especially in artistic subjects; The ultimate intention is that the student acquires, in a practical way, the specific competences related to his training for symbolic communication and the deepening in the graphic analysis of the world around him. This process requires a permanent synthesis of the shapes and forms, colors and lights of objects, to be captured through the skills and graphic methods acquired throughout their academic life. The aim is to exemplify this process with the study and graphic analysis of a work of art, showing the relationship between Industrial Design and humanistic teaching. For this, the work "The Burial of the Count of Orgaz", painted by El Greco, has been chosen, in which there resides a great compositional complexity, full of contrasts and intentionality, but at the same time enormously didactic from the plastic point of view.
\end{abstract}

\title{
Keywords
}

Industrial design · artistic teaching · graphic analysis

- The burial of the Count of Orgaz

\section{La enseñanza de taller en una Escuela de Ingeniería}

La inclusión del Diseño Industrial como disciplina universitaria, dentro de las Ingenierías Industriales, tuvo lugar en España a finales del s. XX. La adaptación de su plan de estudios, bajo las exigencias del nuevo Espacio Europeo de Educación Superior, provocó la reconversión de la primitiva Ingeniería Técnica en el Grado en Diseño Industrial y Desarrollo del Producto, que se fue implantando progresivamente en las diferentes Escuelas de Ingeniería de nuestro país a partir de 2005. 
No obstante, la especificidad intrínseca del Ingeniero de Diseño, que requiere de una potenciación de su capacidad creativa para el ejercicio profesional, así como de una especial formación de corte humanístico y estético, requería de la inclusión de ciertas materias alejadas de las clásicas ofertadas para el resto de Ingenierías. En respuesta a esta necesidad surge la introducción de la enseñanza artística en el ámbito de la Ingeniería, como rasgo distintivo de la titulación de Diseño Industrial, y que recorrerá, de manera transversal, el resto de materias hasta llevar al alumno a una concepción global del proyecto de diseño.

Esta enseñanza tiene como objetivo fundamental, entre otros tantos, la capacitación para la comunicación del alumno mediante lenguajes formales gráficos y simbólicos, que le introduce de lleno en su formación humanística. En efecto, la utilización de un lenguaje gráfico ha representado siempre la expresión de la propia necesidad humana, de igual modo que lo es la experimentación que supone el hecho artístico. Al igual que piensa Park Him (2015, p. 236) para quien la esencia de una educación artística está en el "saber pensar y aprender, saber comunicar, saber hacer, saber relacionar", podemos decir que el hecho gráfico, desde un punto de vista científico, responde a la necesidad de conocer y pensar, de transmitir y comunicarse, de socializar y relacionarse, de pertenecer e identificarse con una comunidad, de ser y existir.

El formato de taller, en el que debe basarse dicha enseñanza, debe reunir las habilidades y conceptos de la expresión artística, para proceder a exponer, en un primer enunciado, una problemática inicial del acto de diseñar, en torno a las funciones del producto, tanto estético-formales (directamente ligadas con la formación previa), así como indicativas y simbólicas, un poco más lejanas pero conectadas, igualmente, a través de la sintaxis visual y la semántica del producto. Se pretende, así, que el alumno aprenda a identificar, formular y resolver problemas, extrayendo datos a partir de los resultados plásticos que experimente para aplicarlos al diseño del producto; ello permite, en definitiva, su introducción paulatina en las fases de desarrollo del mismo a nivel preliminar. 
También debe plantearse la docencia desde una nueva metodología, que permita afrontar primero, mediante un enfoque analítico, una revisión crítica de los conceptos ya aprendidos, ensayados y asimilados, para posteriormente realizar una síntesis que nos lleve a encarar el proyecto de diseño desde su propia génesis plástica. En este contexto, "el enfoque metodológico que, bajo la etiqueta de taller, caracteriza gran parte de las propuestas formativas de algunos contextos de educación no formales permite alejar la Educación Artística de la realización exclusiva de láminas que limitan las posibilidades de las artes visuales para atender a una formación integral del individuo" (Salido López, 2017, p. 352).

\section{Aprendiendo de las fuentes mismas del Arte: "El entierro del Conde de Orgaz"}

Con esta vocación, la revisión que supone la asignatura de Taller de Diseño, en el contexto de los estudios del Grado en Ingeniería en Diseño Industrial y Desarrollo del Producto, se plantea en cuatro bloques diferenciados. El primero, correspondiente al Análisis de componentes y la concepción de forma y volumen, preten- de una actualización y sistematización, como ya se ha visto, de todos los conceptos desarrollados, a nivel más práctico y de manera casi deductiva, en la materia propedéutica de Expresión Artística. En este caso, su desarrollo se estructura en los siguientes temas, de breve y conciso desarrollo:

- Punto y línea.

- El plano y las formas básicas.

- Suprasegmentos gráficos.

- Definición formal y tipos de línea.

- Transformaciones formales.

- Estructura interna de la composición.

- Equilibrio y funciones compositivas.

Los restantes bloques corresponderían al análisis de productos, en sus diferentes funciones, de ese modo, el segundo se destina a sus funciones estético-formales, en una directa aplicación del bloque anterior; el tercero a las indicativas; y finalmente, el último de los bloques, a las simbólicas.

El desarrollo de la asignatura, a la luz del espíritu de los planes de Bolonia, se estructura dualmente. Por un lado, el pro- 
fesor procede a la reflexión de los temas teóricos, a modo de breve explicación en la que abundan los ejemplos visuales, y que rara vez excede los quince minutos de duración; a continuación, se procede, en la mayoría de las ocasiones, a la realización de prácticas de carácter plástico en las que ejercitar esos conceptos expuestos. Por otro lado, también se realiza un trabajo no presencial, que consiste en el desarrollo de cuatro proyectos básicos de diseño del alumno, uno por cada mes de docencia, que sintetizan los conceptos ya ejercitados en el aula, y que, aunque deben ser realizados fuera de este espacio, son convenientemente guiados por el profesor tanto en el horario presencial como en las tutorías, dedicando incluso alguna de las sesiones a su desarrollo.

Es precisamente uno de estos proyectos básicos y aplicados de diseño el que centra nuestro interés, por suponer un aprendizaje directo, a la vez que original e innovador, desde las fuentes mismas del arte. Efectivamente, en este contexto, tal y como menciona Salido López (2017, p. 361), "el acercamiento a la obra de arte y al contexto social y cultural en el que surge favorece el desarrollo del gusto estético, de un pensamiento creativo, del sentido crítico y, a su vez, fomenta actitudes que propician el respeto y la valoración de la manifestación artística en cualquiera de los lenguajes propios de las artes visuales y plásticas".

Con la intención de que sirva para la aplicación de todos los conceptos desarrollados en el primer bloque, anteriormente enumerados, el paso inicial correspondería a la selección, por parte del alumno, de una obra de arte bidimensional, ya sea una pintura o una fotografía reconocida; a partir de la misma, se pretende que el alumno realice un análisis exhaustivo en cuanto a unos parámetros determinados, ya desarrollados a lo largo de las clases teóricas, y que le permitirá la realización coherente de dos diseños: el primero de ellos, un marco para la obra elegida, a modo de síntesis de todos los análisis realizados; el segundo, un objeto, de libre temática, concepción y ejecución, que condense el desarrollo conceptual de la obra. El estudio a desarrollar, a partir de la imagen elegida, consta de las siguientes fases, para las que no se fija un número determinado de análisis, pudiéndose aunar varias de esas representaciones en un único resultado:

- Análisis de la definición formal de los elementos de la obra mediante contornos. 
- Interpretación de la génesis formal a partir de las formas básicas y sus transformaciones.

- Análisis del peso visual y las fuerzas en la composición.

- Representación esencializada del color y la luz.

- Análisis general de la composición.

La explicación de la metodología utilizada seguirá el guión gráfico desarrollado por uno de los autores, quien eligió para su análisis el cuadro de "El entierro del Conde de Orgaz" (fig. 1), la obra maestra de entre todos los cuadros pintados por Doménikos Theotokópoulos (15411614), conocido como "El Greco" en referencia a su origen. Está ubicado desde su ejecución en Toledo, en la iglesia de Santo Tomé, por cuyo párroco fue encargado (apareciendo retratado en el eclesiástico que oficia la ceremonia), como reza una placa junto a la obra (Perales Soriano, 1978, p. 38): “Dominico Greco, pintó el a cuadro del Entierro del Conde de Orgaz por encargo de don Andrés Núñez, Párroco de esta Iglesia de Santo Tomás Apóstol. Se firmó el contrato el día dieciocho de marzo de MDLXXXVI".

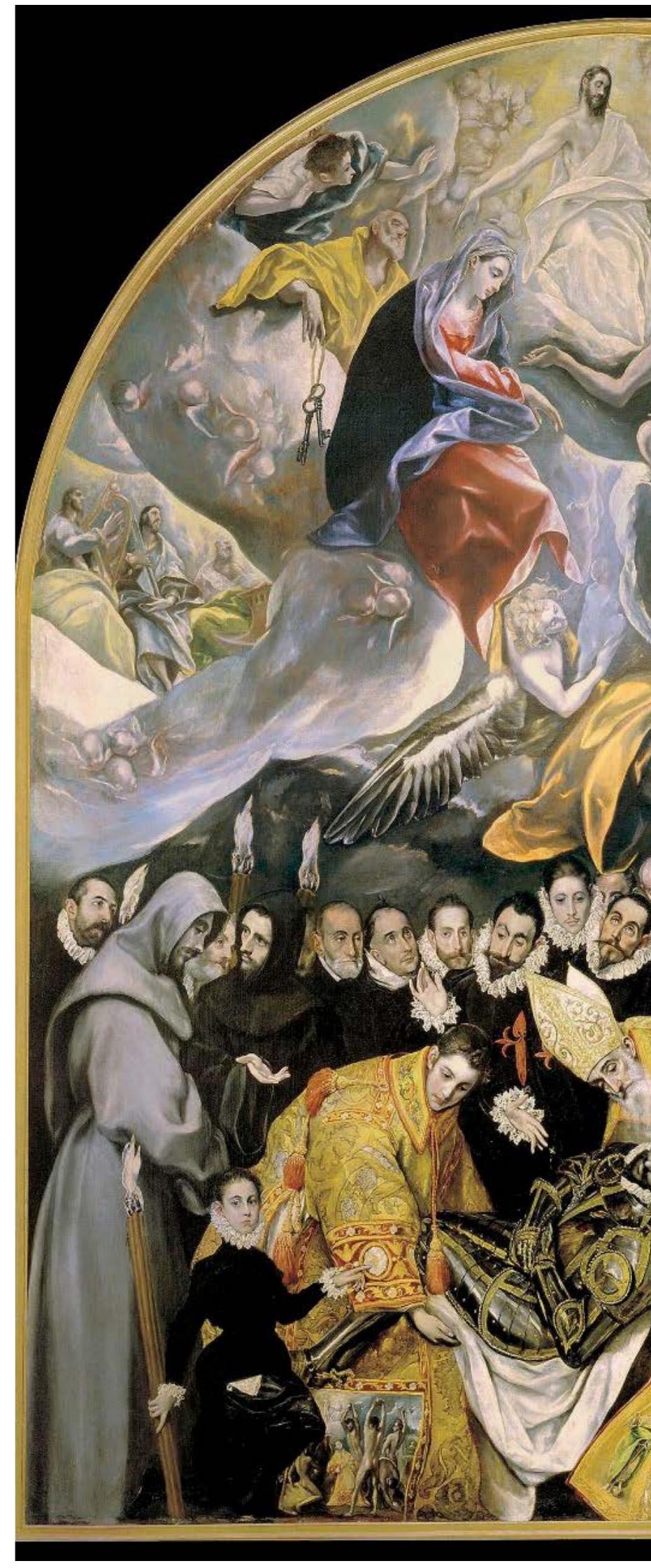

Fig. 1. El Entierro del Cond 


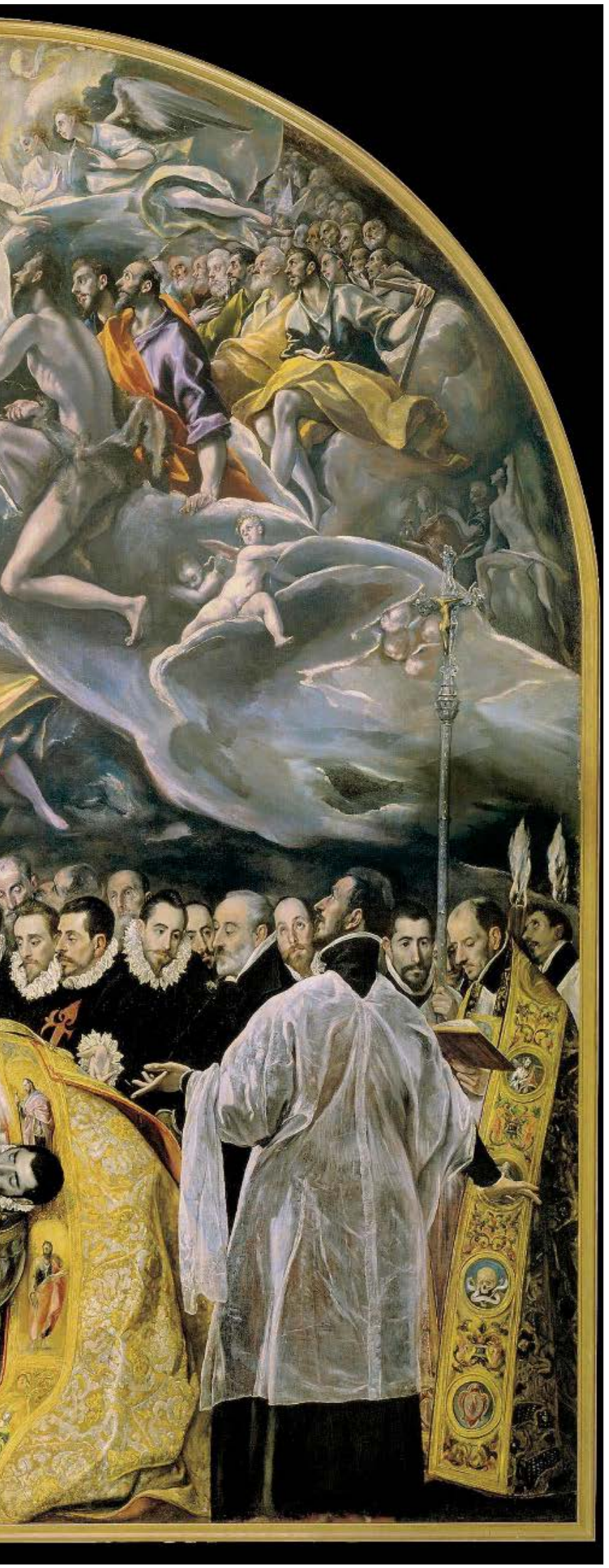

e de Orgaz. El Greco. 1587.
La obra se vincula a la biografía de D. Gonzalo Ruiz de Toledo que, a pesar de la popular denominación del cuadro, no era conde, sino señor de Orgaz. Emparentado con las familias que ostentaban el poder en Castilla entre los siglos XIII y XIV, era uno de los nobles más apreciados de Toledo, y destacaba por una piedad y espíritu devoto que le llevaron a ser benefactor de fundaciones, templos y órdenes religiosas. Murió el 9 de diciembre de 1323, acaeciendo en sus pompas fúnebres un milagro, según la tradición, que recoge la reproducción de la placa original dispuesta junto al cuadro, en el zócalo (Perales Soriano, 1978, p. 36):

“Don Gonzalo Ruiz de Toledo, Señor de Orgaz, Notario Mayor de Castilla entre las muchas obras de piedad a la Iglesia de Santo Tomás Apóstol (...), en la que por testamento mandó ser enterrado que en otro tiempo era reducida y mal conservada dispuso fuese ampliada a mayor espacio a costa de su dinero donando a este fin muchos recursos en plata y oro. Mientas los sacerdotes se preparaban para inhumarlo sucedió el hecho admirable e insólito, San Estaban y San Agustín bajando del cielo con sus propias manos aquí lo colocaron en el sepulcro (...). Murió en el año del Señor 1322". 
El Greco consiguió un resultado magistral, considerada la obra por muchos como una de las cumbres de la historia de la pintura, pero siendo muy fiel a las especificaciones y elementos que se fijaban en el contrato formalizado en 1586: "En lo de más abajo... se ha de pintar una procesión de cómo el cura y los demás clérigos que estaban haciendo los oficios para enterrar a don Gonzalo de Ruiz de Toledo, Señor de la villa de Orgaz, y bajaron san Agustín y san Esteban a enterrar el cuerpo de este caballero, el uno teniéndole la cabeza y el otro los pies echándole en la sepultura y fingiendo alrededor mucha gente que estaba mirando y encima de todo esta se ha de hacer un cielo abierto de gloria..." (Brown, 1982, p. 124).

\section{La definición formal mediante líneas de contorno}

El primer nivel del estudio corresponde, por tanto, a la definición formal de los elementos de la obra, que permite su diferenciación del continuo gráfico. En este punto, merece la pena recordar la distinción señalada por Arnheim (1980), entre las dos acepciones de la palabra española "forma": por un lado, la "shape" inglesa haría referencia únicamente a la forma material, visible y palpable; por otro, la "form" aludiría a la configuración, abarcando ya lo estructural y lo no directamente observable por los sentidos. Refiriéndonos, en este caso, a la primera de las acepciones en exclusiva, este primer análisis, por tanto, consistiría en la identificación y plasmación de los elementos menores que configuran la forma y la cualifican.

En esa definición formal, el elemento plástico más simple que se puede emplear, y a la vez más hegemónico, es la línea, como generadora del plano y, por tanto, de la forma. Siguiendo a Arnheim (1980), podríamos clasificar los tipos de líneas en tres diferentes clases:

- La línea objetual, que permite apreciarla en su naturaleza unidimensional, manteniendo su independencia en sus uniones y combinaciones, que tienen lugar según la ley de la simplicidad. 
- La línea de sombreado que, en agrupación y combinación con otras, forma una superficie, dejando de ser un objeto individual.

- La línea de contorno, que deja de ser un objeto unidimensional independiente para transformarse en contorno delimitador de un objeto bidimensional; se convierte así en parte de un todo, y que segrega un elemento de ese todo, encerrando en sí la parte del espacio que le corresponde.

En efecto, la identificación de la existencia de un perfil o arista perimetral, en definitiva, de un contorno, es un medio básico para la identificación de la forma como apariencia externa, para su definición en el espacio. Como mencionó Gubern en 1996:

"cuando de las rayas aleatorias surgió para su productor (o para un observador) un sentido, una imagen, haciendo de la línea un significante, se asistió propiamente a lo que René Thom Ilamó una catástrofe, es decir, un salto cualitativo del caos al sentido. Y el posterior control intencional de la línea como significante de la producción icónica la convirtió literalmente en un símbolo".

El análisis de contornos (fig. 2) nos lleva a la primera evidencia compositiva del cuadro: la especial configuración del emplazamiento originario, en la capilla funeraria donde fue enterrado el señor de Orgaz, con una parte cuadrada y otra semicircular, hacía posible la división, de gran significado teológico, entre una parte terrenal (la cuadrada) y otra parte celestial (la semicircular), ya enunciada en el contrato de ejecución. 


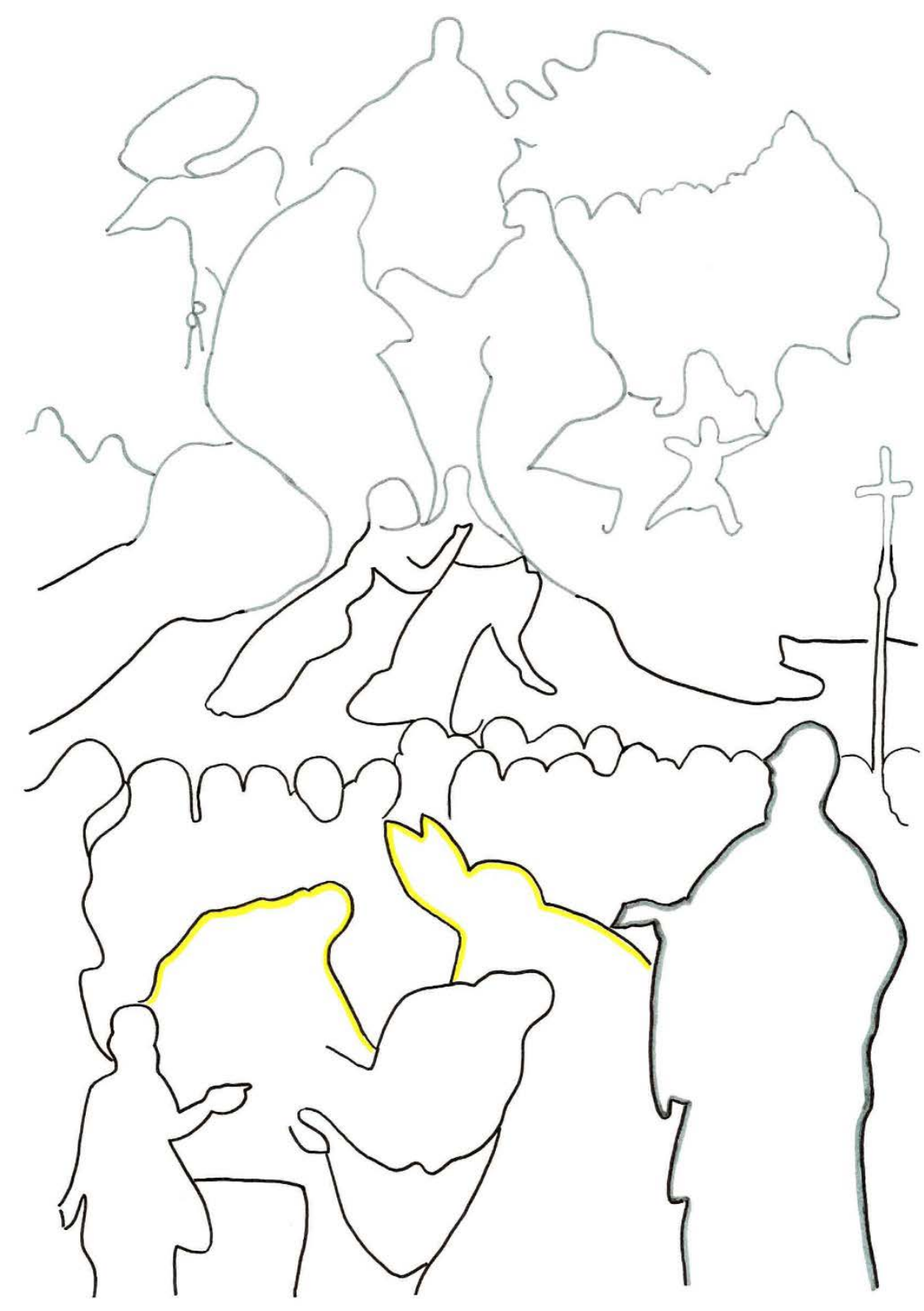

Fig. 2. Análisis formal a partir de contornos de "El Entierro del Conde de Orgaz". Elaboración propia.

Los contornos, pues, de ambas realidades, se grafían de manera distinta según su naturaleza: los de los personajes que asisten al entierro, en una escena dominada por el luto y la seriedad, se marcan en color negro; para los contornos de las entidades celestiales, de carácter más etéreo, se utiliza el color gris. Son dos los únicos elementos que comparten ambos colores para sus respectivos contornos, puesto que se ubican 
en un espacio compartido entre las dos realidades del cuadro: por un lado, el ángel que transporta el alma del conde, y cuya presentación a la corte celestial entre las nubes supone el punto central de la composición; por otro lado, la cruz procesional, de evocador simbolismo, como representación de la realidad celestial en la parte terrestre.

Asimismo, los contornos se degradan, en una valoración de la línea en directa analogía con la importancia de los personajes: los correspondientes a las figuras de primer plano o principales de la composición se individualizan, mientras que los restantes recogen agrupaciones por cercanía o similitud. De esta valoración emergen ya tres personajes en la parte más cercana al espectador: las dos figuras celestiales artífices del milagro, los santos Esteban y Agustín, revestidos en el cuadro con ropas doradas, y cuyo contorno se refuerza en el análisis con ese mismo color; también se singulariza, con un mayor grosor, el contorno del cura con roquete, que dando la espalda al público y haciendo caso omiso del entierro, observa con detenimiento la entrada del alma en la esfera celestial.

\section{Génesis formal, peso visual y fuerzas en la composición}

El segundo nivel, pues, correspondería a una interpretación de la génesis formal, en los dos planos ya señalados por Arnheim (1980), a partir de las formas gráficas y sus consiguientes transformaciones. Así pues, y considerando la forma de un objeto físico situado en un espacio como una descripción geométrica de la parte del espacio ocupada por él, la delimitación de su límite exterior, y su explicación a partir de la transformación dimensional, aditiva o sustractiva de una o varias formas básicas, es de sumo interés para describir los procesos compositivos que se dan, y que serán analizados en los siguientes niveles. Todo ello, sin tener aún en cuenta su ubicación y orientación en el espacio, su tamaño ni ninguna otra propiedad como el color, el contenido y la composición del material, que nada tienen que ver con su naturaleza formal.

La concepción de las formas básicas partiría de la teoría enunciada por Kandisnky (1952), según la cual se establecen correlatos entre el ángulo, originado por una línea quebrada, y su evolución hacia la forma cerrada. Así, el triángulo se asocia con el ángulo agudo, transmitiendo 
equilibrio, jerarquía y unión; el cuadrado, en relación directa con el ángulo recto, se considera la forma más objetiva y, por ello, trasmite equilibrio y estabilidad; el círculo, por último, se asocia con el ángulo obtuso (y éste con la génesis de la curva), siendo el paradigma de la continuidad, el movimiento y el dinamismo.

Por su parte, y como dice Ching (1979), en efecto, "cualquier elemento formal es susceptible de ser percibido como una transformación de las formas básicas, mediante la manipulación de sus dimensiones o de la adición o sustracción de otros elementos". Así, las transformaciones dimensionales, que explican la irregularidad, suponen una modificación, mediante ampliación o reducción, de alguno de los parámetros de la forma para crear un elemento diferente; las transformaciones aditivas son aquellas que obtienen nuevos elementos formales mediante la combinación de otros tantos, tanto regulares como irregulares; por último, las transformaciones sustractivas son las que explican la génesis de una nueva forma a través de la sustracción de ciertos elementos a otro primigenio, dependiendo del alcance de esta sustracción la conservación de su identidad original.

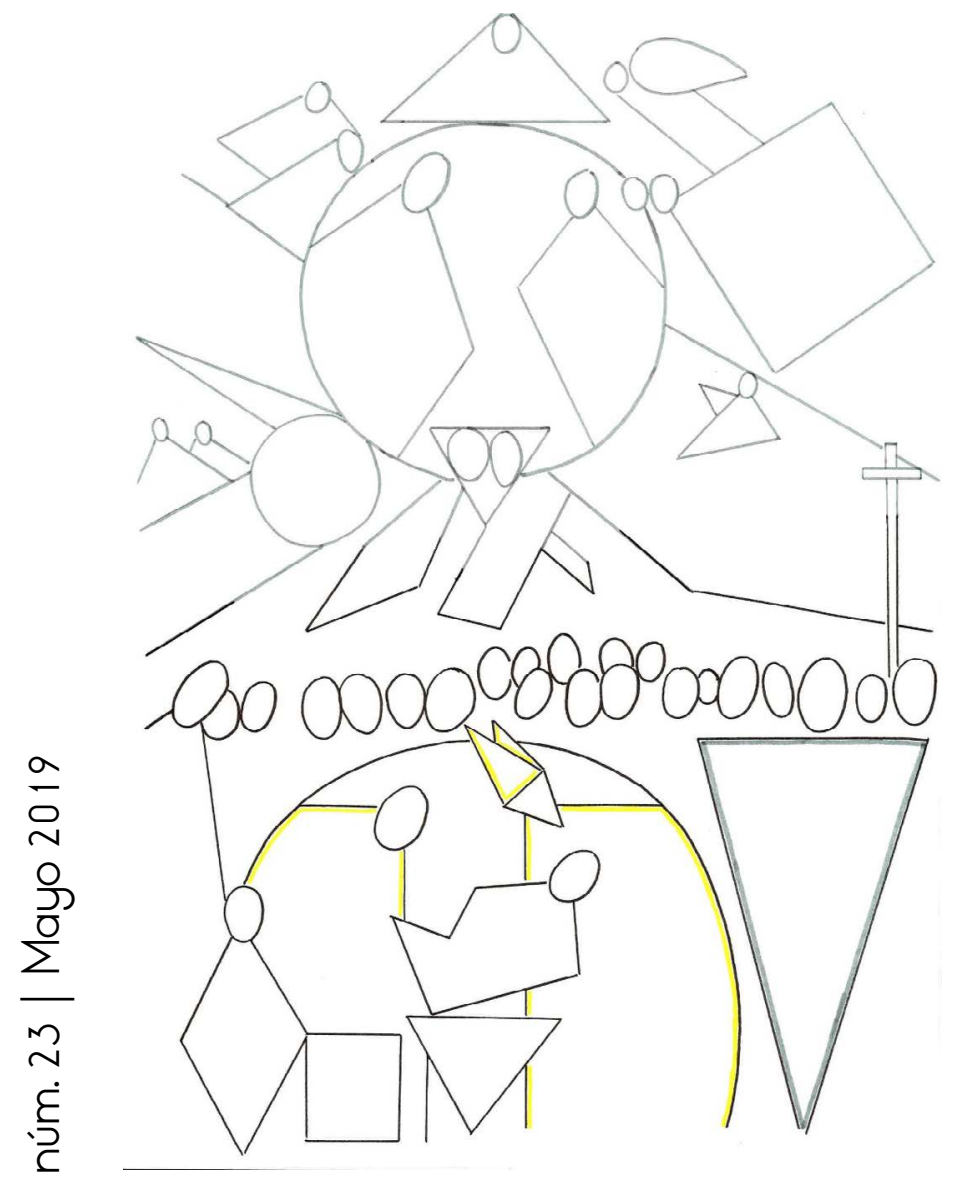

Fig. 3. Génesis a partir de formas básicas y análisis del peso visual de "El Entierro del Conde de Orgaz". Elaboración propia. 
La segunda composición analítica (fig. 3) comprende la interpretación de la génesis formal, que incluso se adentra, por su pertinencia, en el terreno de la configuración o estructura, y el análisis del peso $y$ las fuerzas visuales. El peso visual determinaría, en última instancia, el poder de atracción gravitatoria de cada elemento de la composición. Es una fuerza visual inseparable de los objetos, una cualidad real e intrínseca a su propia naturaleza, pero que difícilmente puede medirse, debido a la multiplicidad de factores que en él influyen. Sin embargo, también pueden existir otro tipo de fuerzas que pueden actuar en la composición, con independencia de su origen o del elemento al que afectan.

La esencialización de las entidades del cuadro en formas básicas, por tanto, unida a la focalización de la acción en la pareja de escenas principales, circunscritas en dos grandes formas circulares más conceptuales que reales, muestran una diferenciación también compositiva en la dualidad anteriormente comentada. Así, la zona terrenal se desvela como una composición muy lineal, regida por la agrupación de formas circulares que representan las cabezas de los caballeros que, en abultado número, asisten al fune- ral con desigual atención; la composición celestial, por el contrario, se muestra en clara disposición centrífuga. La fuerza visual de un triángulo invertido representa al único personaje que participa, a la vez, de las dos realidades descritas. El peso visual de la composición bascula en el eje que une las dos escenas principales $y$, más concretamente, las cabezas de Cristo y el conde, pasando por el alma. La diferente tonalidad de líneas que ya veíamos en el análisis de contornos se mantiene.

\section{Esencialización del color y la luz.}

El tercer nivel de análisis tiene como objetivo el conseguir una representación esencializada del color y la luz. En este sentido, Kandinsky (1923) ya apuntaba que "el color debe ser estudiado, igual que cualquier otro fenómeno, desde diferentes puntos de vista, con diferentes orientaciones y con los correspondientes métodos"; así, en este nivel, la teoría del color interesa por su estudio de la realidad cromática en tanto que está siempre ligada a la forma, incluso, según Wick (1988), configurándola, concretándola y modificándola. Para Kandinsky (1952), "ninguna superficie ni ningún espacio puede existir sin color", puesto que el co- 
lor está siempre ligado a la forma. Asimismo, y en cuanto a su uso en el arte, el color representa además una función expresiva de gran importancia, resaltando aquellas partes de la composición gráfica que el artista considera más relevantes, con una clara función localizadora.

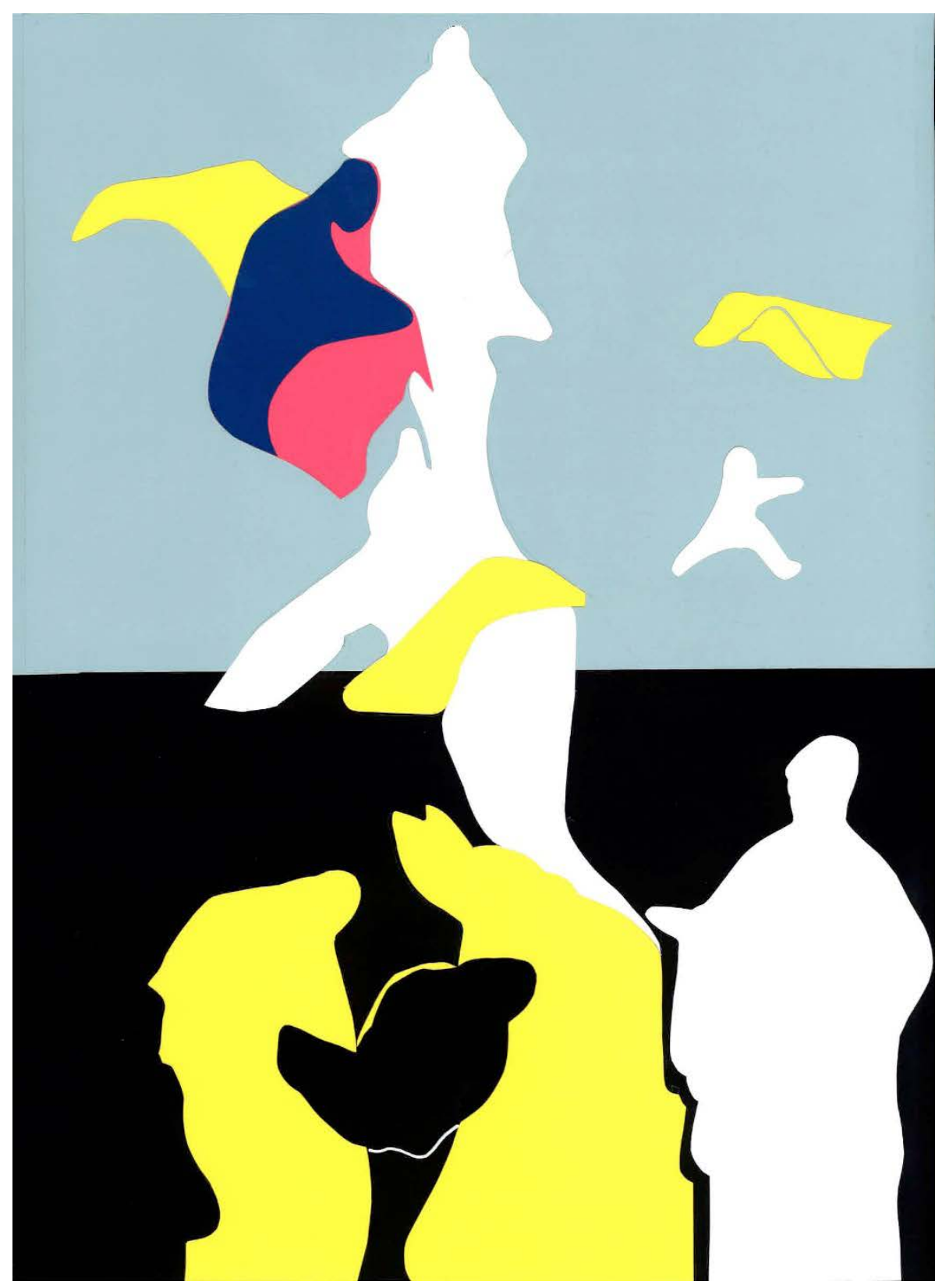

Fig. 4. Análisis del color en "El Entierro del Conde de Orgaz". Elaboración propia.

En el estudio del color (fig. 4), la dicotomía entre cielo y tierra, también presente en los anteriores niveles, se refleja en el uso de una tonalidad negra para la parte terrestre y otra azul para la celeste. Sobre el fondo negro en el que se desarrolla el entierro, 
se singularizan de dorado los dos santos protagonistas del milagro que se relata, $y$ que abrazan el cuerpo, recortado en negro sobre sus siluetas. El eclesiástico con roquete (en el que se retrata al ecónomo parroquial Pedro Ruiz Durón), que participa con su mirada de la escena celestial, se representa, por su importancia y su intencionada iluminación, con una silueta de color blanco recortada sobre el fondo. De la escena central emana un halo blanco, que remarca la trayectoria que recorre el alma del conde hasta conectarlo con el mismo Cristo. En la zona celeste, sobre el fondo neutro azulado, frío pero intenso, que subsume la naturaleza etérea de las entidades representadas, se singularizan, mediante un color dorado, los ropajes con que se visten determinados personajes destacados (San Pedro y Santo Tomás), así como el ángel que lleva el alma del difunto a su última rendición de cuentas. Además, aparece remarcada, en los colores que la tradición le asigna, la imagen de la Virgen María, única figura con una gama cromática completamente diferente al resto del cuadro.

Por su parte, la luz, desde un punto de vista meramente fisiológico, es una condición indispensable para que en nuestro ojo se pueda estimular la percepción del hecho visual. Pero ya en un plano compositivo, un poco más trascendente, la iluminación tiene tres funciones en los lenguajes visuales: en primer lugar, aportar unidad y jerarquía en el continuo gráfico, matizando las partes para igualarlas en un todo; por otro lado, una luz puntual enfatiza determinados elementos formales para separarlos y diferenciarlos del todo, singularizándolos; por último, la luz aporta a las dos dimensiones la noción de volumen, creando la sensación del espacio, la tercera dimensión, a partir del plano.

La existencia de la luz en un espacio ocupado implica la aparición de la sombra, que se establece al interponer un objeto en la trayectoria de propagación de la emisión luminosa, ayudando a establecer una jerarquía entre los diferentes elementos. Estas sombras pueden diferenciarse en dos tipos diferentes que, aun producidos en aquellas zonas donde hay poca luz $y$, por tanto, con un mismo origen, perceptualmente emergen como conceptos muy distintos. La sombra propia es una alteración de la incidencia de la luz sobre el propio objeto debida a su propia forma intrínseca, y colabora en la definición de su volumen; la sombra arrojada o esbatimentada es aquella que surge por la interposición de un objeto 
en la trayectoria de iluminación de otro, definiendo su posición en el espacio y en relación con otros elementos.

Para el análisis de la luz (fig. 5), se partió, como estrategia plástica fundamental, de un fondo negro, que implicaría la total oscuridad, para marcar sobre él aquellas zonas o puntos iluminados. Jesucristo ocupa el lugar con más luz del cuadro, vestido de forma gloriosa, de blanco, como juez de vivos y muertos, y ordenando a San Pedro abrir las puertas del Cielo para la piadosa alma del conde. La efusión de luz baja, desde ese punto superior, de manera vertical, hasta el cuerpo del conde, pasando por el alma; destaca, por su importancia, la iluminación concreta de esta entidad "bisagra", en la intención de unir las dos realidades ya que, partiendo de la tierra misma, se dispone a entrar en los cielos. En la zona terrenal, la luz proviene desde la parte izquierda del cuerpo, dejando en sombra el costado derecho de todos los personajes representados. Dada la especial configuración de la parte baja, donde predomina el color negro, no aparecen representadas sombras arrojadas. 


\section{Análisis general y desarrollo de un marco y un objeto}

Los cuatro niveles de análisis deben llevar al alumno a una esencialización de la composición en una suerte de estudio general, en el que deberán primar aquellos rasgos o aspectos más sobresalientes y conclusivos de los análisis parciales. Esta composición final deberá presentar las conclusiones o resultados de manera rotunda pero, a la vez, dejando el terreno de la objetividad para adentrarse en el plano más subjetivo e interpretativo, relanzando el conocimiento, emanado de las fuentes mismas del arte, hacia la creatividad que supone el proyecto de diseño.

El análisis general final presentado (fig. 6) pretende condensar, por tanto, de la manera más esencial posible, los hallazgos más importantes de los anteriores análisis. Un gran triángulo, configurado por una línea blanca de grosor ciertamente relevante, une los tres puntos que se consideran claves: la cabeza del conde, por centrar la escena terrenal, la figura de Jesucristo, del que emana la majestad de la parte superior, y los ojos del cura con roquete, que conecta con su mirada las dos realidades. En la parte celeste aparecen otras líneas blancas, que marcan el 
contorno de la organización piramidal según la cual se dispone la corte de personajes; una composición de puntos simboliza la presencia del casi infinito grupo de los bienaventurados, hasta este análisis obviado, que comparten con Cristo ese lugar de privilegio. Dos simples curvas simbolizan, por un lado, a la Virgen (con sus colores propios), y por otro, la trayectoria ascendente del alma del conde, en una tonalidad blanquecina que destaca sobre el fondo azul.

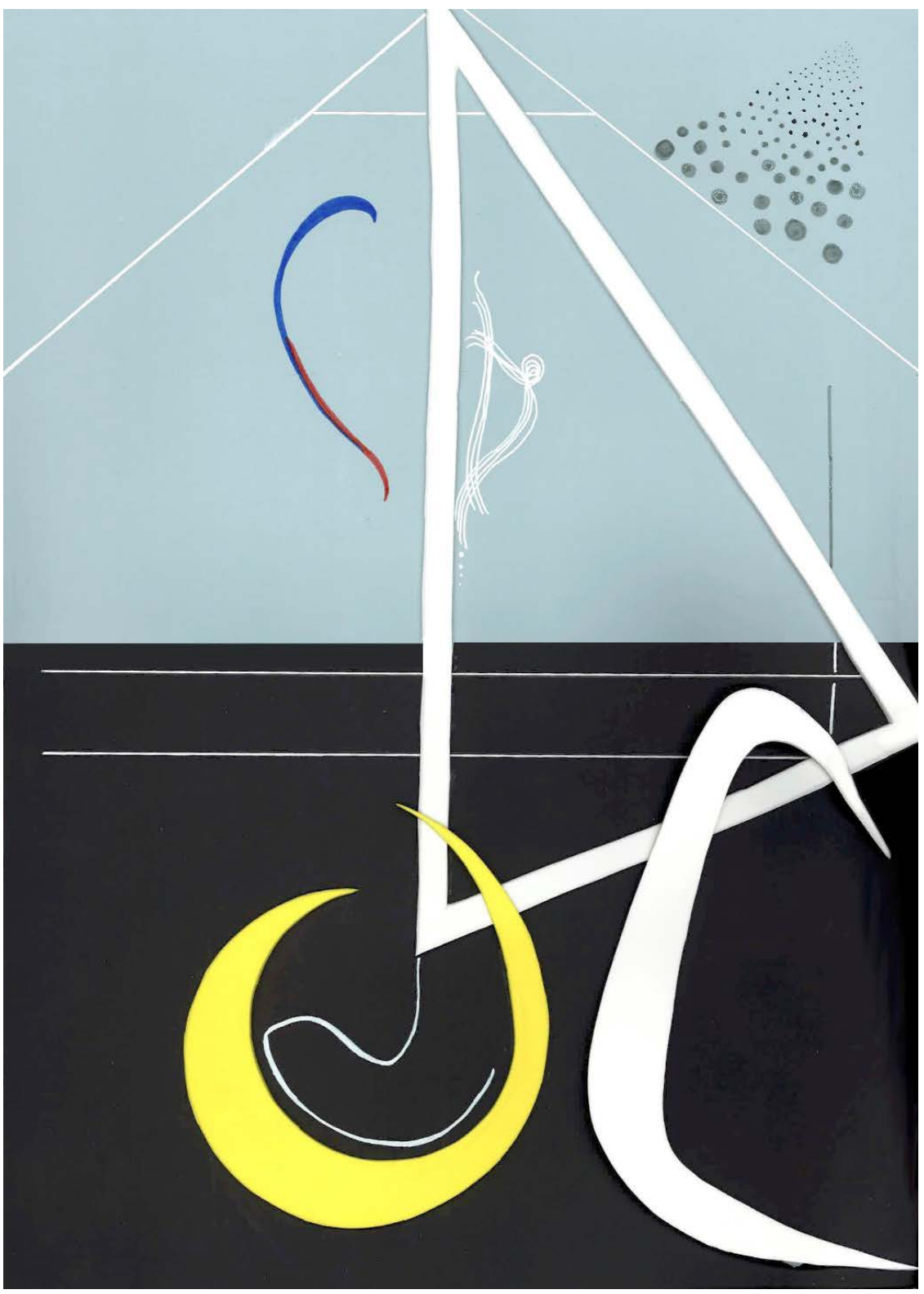

Figura 6. Análisis final de "El Entierro del Conde de Orgaz". Elaboración propia. 
Por su parte, en la parte terrestre, dos únicas líneas blancas de muy reducido grosor, destacando sobre el fondo negro, marcan la disposición en dos filas de los asistentes al entierro, contrapuestas a la verticalidad que impone otra línea que simboliza la cruz procesional. La escena central se simboliza con una curva envolvente dorada, en clara alusión a la coloración de los ricos ropajes del cuadro, que parece acunar la línea blanca que representa el cuerpo sin vida del conde. El eclesiástico dispuesto de espaldas se representa mediante otra curva, en este caso blanca, sobre la que reposa el triángulo compositivo principal.

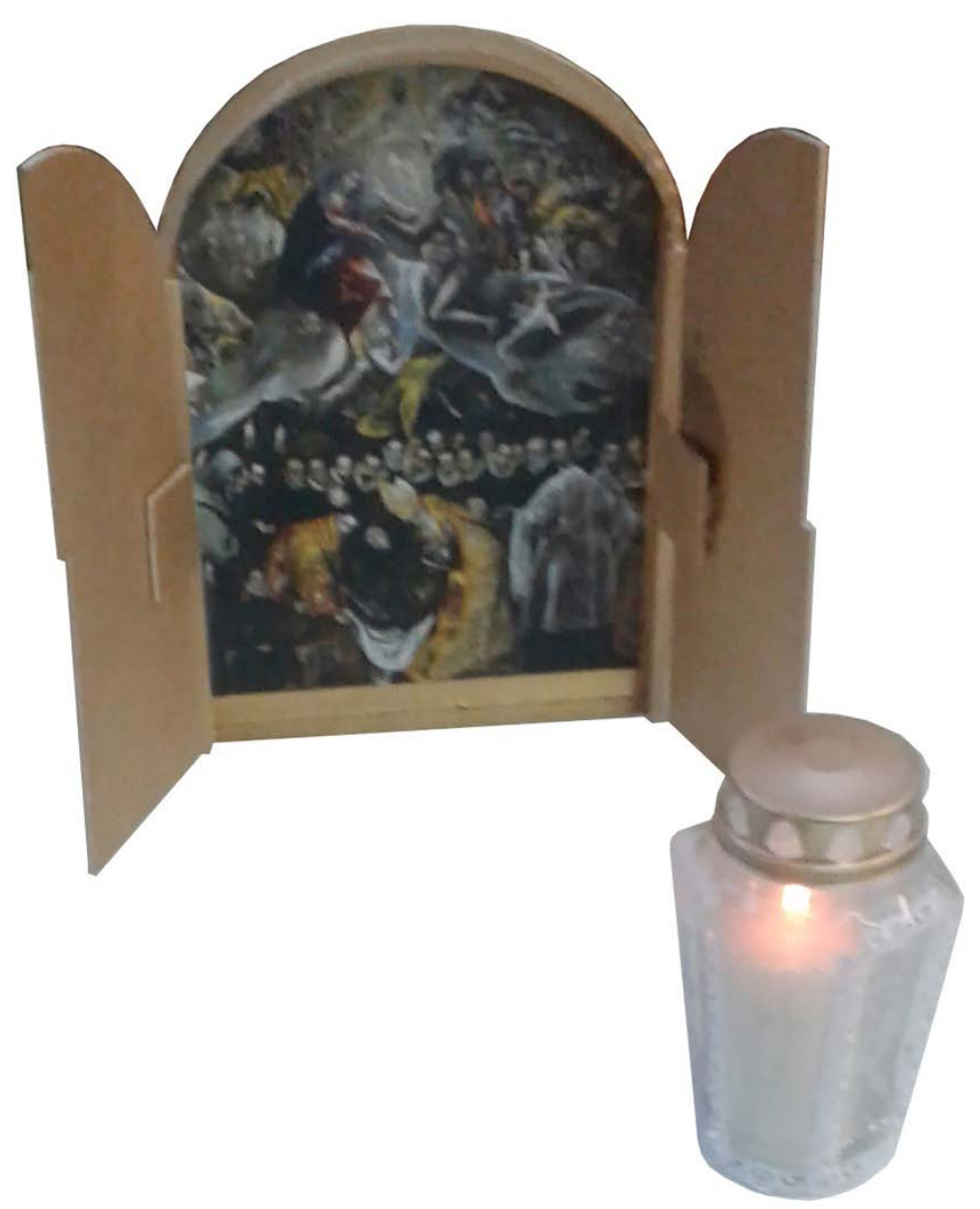

Fig. 7. Diseño de marco y objeto a partir del análisis de "El Entierro del Conde de Orgaz".

Por último, y como se mencionó con anterioridad, el trabajo debía cristalizar en el diseño de un marco para el cuadro y de un objeto que condensase la idea principal del mismo (fig. 7). La concepción del marco partió de su lugar de exposición, que condiciona la forma del bastidor, ocupando toda una pared en el interior de la iglesia; así, se 
diseñó como una ventana, a modo de retablo, mueble, altar portátil o tríptico, y cuyas puertas, con la máxima simplicidad, aparecen partidas en dos partes, en clara alusión a las dos realidades en las que se divide la obra. El cuadro pasa así, de una condición como elemento perteneciente a un inmueble, por su intrínseca implantación en la iglesia, a considerarse como elemento transportable.

Por el contrario, el objeto de diseño se ideó desde un punto más metafísico, en contraposición a esa concepción tan material de la composición en cuanto al marco. En este sentido, la omnipresencia en la práctica totalidad de los análisis del halo blanco que une, de manera vertical y rotunda, el cielo (en la figura de Jesucristo) y la tierra (representada en el cuerpo inerte del Conde), pasando por el momento congelado en que el alma, en brazos del ángel, penetra en el cielo, hizo que la idea principal del cuadro se materializara en una vela, en la que brilla una llama, y que, al apagarse, emite un humo en forma de hilo blanquecino...

\section{A modo de conclusión...}

La convergencia de los estudios universitarios en el Espacio Europeo de Educación Superior ha supuesto la introducción de nuevas maneras de enseñar, el desarrollo de nuevas metodologías y recursos para afrontar el aprendizaje y la necesidad de una innovación en la docencia para la adquisición, por parte del estudiante del siglo XXI, de la relación de competencias que contemplan los nuevos planes de estudio.

A esta realidad no es ajena la disciplina del Diseño Industrial, surgida a finales del pasado siglo con el fin de sistematizar, a nivel universitario, una atribución profesional que venía siendo desarrollada por técnicos de la más diversa procedencia. La Ingeniería Técnica en Diseño Industrial y Desarrollo del Producto, y su correspondiente grado desarrollado a la luz del EEES, están englobados en la rama de las Ingenierías Industriales, pero con ciertas especificidades en sus planes de estudios que adentran su docencia en el campo de las humanidades. La formación artística es, por tanto, un rasgo específico de las mismas, que forma al alumno en competencias relacionadas con la comunicación en lenguajes gráficos y simbólicos. 
La capacitación en la comunicación gráfica, por tanto, se debe fundamentar en el conjunto de capacidades físicas y cognitivas que comprenden su materialización, percepción y descodificación. Además, su aprendizaje supone un tipo especial de conocimiento, pues implica haber interiorizado, de manera no consciente, un determinado conjunto de pautas y de principios. Por otro lado, su utilización representa una forma de actividad que se desarrolla en un medio social y para alcanzar determinados objetivos que dependerán de la situación concreta. Por último, el conocimiento que aporta debe dar lugar a productos concretos, puesto que la realidad directamente observable está constituida por muestras gráficas.

Desde este punto de vista, el desarrollo de su docencia y aprendizaje puede ser entendido mediante la puesta en marcha de una clase en formato de taller; de esta manera se alimenta la colaboración directa entre el alumno y el profesor, a través de la práctica directa, y preferiblemente desde las fuentes mismas del arte. Las enseñanzas que, solo así, se pueden extraer, representan un compendio de habilidades y destrezas, directamente experimentadas por el alumno, que complementan el corpus teórico necesariamente inherente a su propia formación.

\section{Bibliografía}

Arnheim, R. (1980). Arte y percepción visual: Psicología del ojo creador. Balseiro, M. L. (trad.). 14a edición. Madrid, España: Alianza Editorial, 1997. 553 p. Alianza Forma; 3. ISBN: 8420670030.

Brown, J. (1982). El Greco de Toledo. Madrid, España: Alianza Editorial, 1982. 280 p. ISBN: 8420690155.

Ching, Francis D. K. (1979). Arquitectura: forma, espacio y orden. $3^{\text {a }}$ edición. Barcelona, España: Gustavo Gili, 1997. ISBN: 9788425223440. 
Gubern, R. (1992). La mirada opulenta: Exploración de la iconosfera contemporánea. Barcelona, España: Gustavo Gili, 1992. ISBN: 9789688871973.

Kandinsky, W. (1923). "Farbkurs und Seminar". En: Gropius, W. (ed.): Staatliches Bauhaus in Weimar 1919-1923. Weimar-Munich, Alemania: Bauhaus-Verlag, 1923. p. 27.

Kandinsky, W. (1952). Punto y línea sobre el plano: contribución al análisis de los elementos pictóricos. Echevarren, R. (trad.). $1^{\text {a }}$ edición. Barcelona, España: Paidós Ibérica, 1952-1998. 159 pp. Paidós Estética; 25. ISBN: 8449303141.

Park Him, D. H. (2015). "La educación artística como elemento clave en el proceso de aprendizaje en escuela". En: Heras Evangelio, D; Rodríguez Calatayud, N. (2015). Ilustrafic, $2^{\circ}$ Congreso Internacional de llustración, Arte y Cultura Visual: Libro de Actas. pp. 226-237. Valencia, España: Editorial universitat Politècnica de València, 2015. ISBN: 97884-9048-314-5. DOI: http://dx.doi.org/10.4995/ILUSTRAFIC/ILUSTRAFIC2015/1125.

Perales Soriano, G. (1978). "Apunte histórico y técnico sobre la nueva instalación del Entierro del Conde de Orgaz". En: El Entierro del Conde de Orgaz: nueva instalación estudio científico y tratamiento. Informes y trabajos del Instituto de Conservación Restauración de Obras de Arte, 13. Ministerio de Cultura. Dirección General del Patrimonio Artístico, Archivos y Museos. Madrid, España: Instituto de Conservación y Restauración de Obras de Arte, 1978. ISBN: 84-500-2725-X.

Salido-López, P. V. (2017). "La Educación Artística en el contexto de las competencias clave: del diseño a la evaluación de talleres didácticos en la formación de formadores". En: Arte, Individuo y Sociedad, 29(2), pp. 349-368. Madrid, España: Universidad Complutense de Madrid. ISSN: 1131-5598. DOI: http://dx.doi.org/10.5209/ARIS.54655

Wick, R. (1988). La pedagogía de la Bauhaus. Bas Álvarez, B. (trad.). Madrid, España: Alianza Editorial, 1988. 317 pp. Alianza Forma; 54. ISBN: 8420670545. 


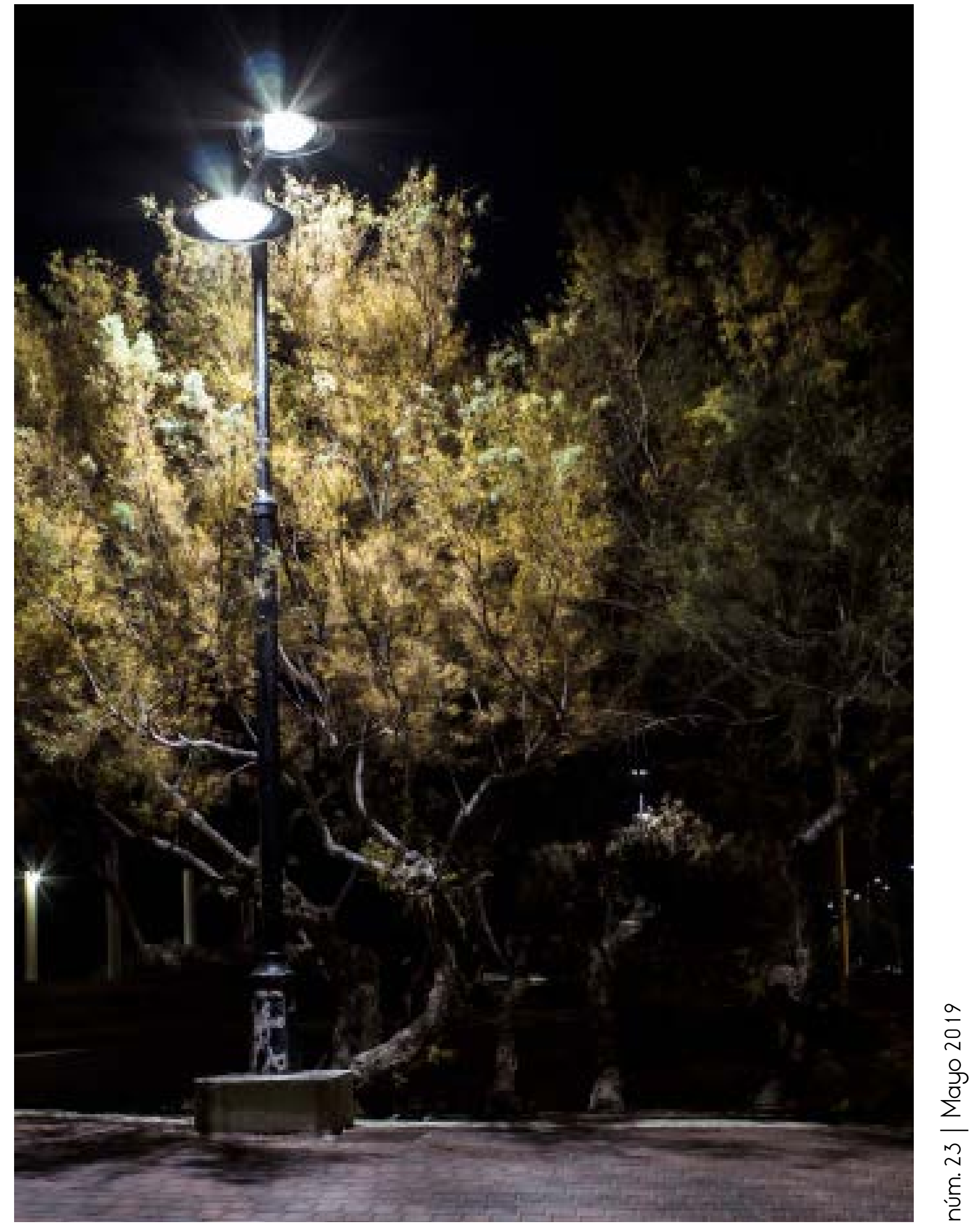

